\title{
PSCA is a target of chimeric antigen receptor T cells in gastric cancer
}

\author{
Di Wu $u^{1,2,3}$, Jiang $\mathrm{Lv}^{2,3,4}$, Ruocong Zhao ${ }^{2,3,5}$, Zhiping $\mathrm{Wu}^{2,3,4}$, Diwei Zheng ${ }^{2,3,4}$, Jingxuan Shi ${ }^{2,3,4}$, Simiao Lin ${ }^{2,3}$, \\ Suna Wang ${ }^{2,3}$, Qiting Wu ${ }^{2,3}$, Youguo Long ${ }^{2,3}$, Peng Li, ${ }^{2,3,6^{*}}$ (i) and Yao Yao ${ }^{2,3^{*}}$
}

\begin{abstract}
Background: Gastric cancer is a deadly malignancy and is a prognostically unfavorable entity with restricted therapeutic strategies available. Prostate stem cell antigen (PSCA) is a glycosylphosphatidylinositol (GPI)-anchored cell surface protein widely expressed in bladder, prostate, and pancreatic cancers. Existing studies have thoroughly recognized the availability of utilizing anti-PSCA CAR-T cells in the treatment of metastatic prostate cancer and nonsmall-cell lung cancer. However, no previous study has investigated the feasibility of using anti-PSCA CAR-T cells to treat gastric cancer, irrespective of the proven expression of PSCA on the gastric cancer cell surface.

Methods: We determined the expression of PSCA in several primary tumor tissues and constructed thirdgeneration anti-PSCA CAR-T cells. We then incubated anti-PSCA CAR-T cells and GFP-T cells with target tumor cell lines at E:T ratios of 2:1, 1:1,1:2, and 1:4 to evaluate the therapeutic efficacy of anti-PSCA CAR-T cells in vitro. We also assayed canonical T cell activation markers after coculturing anti-PSCA CAR-T cells with target cell lines by flow cytometry. The detection of a functional cytokine profile was carried out via enzyme-linked immunosorbent assays. We then evaluated the antitumor activity of anti-PSCA CAR-T cells in vivo by establishing two different xenograft GC mouse models.
\end{abstract}

Results: Anti-PSCA CAR-T cells exhibited upregulated activation markers and increased cytokine production profiles related to T cell cytotoxicity in an antigen-dependent manner. Moreover, anti-PSCA CAR-T cells exhibited robust anti-tumor cytotoxicity in vitro. Importantly, we demonstrated that anti-PSCA CAR-T cells delivered by peritumoral injection successfully stunted tumor progression in vivo. However, intravenous administration of anti-PSCA CAR-T cells failed to reveal any therapeutic improvements.

Conclusions: Our findings corroborated the feasibility of anti-PSCA CAR-T cells and their efficacy against gastric cancer, implicating the potential of applying anti-PSCA CAR-T cells to treat GC patients in the clinic.

Keywords: Chimeric antigen receptor T cells, Gastric cancer, Prostate stem cell antigen, Immunotherapy

\section{Introduction}

Gastric cancer is a significant public health issue, as the fourth most common cancer and the third leading cause of cancer death worldwide [1]. The overall patient survival is significantly poor because of late diagnosis and suboptimal therapies. For early diagnosed patients, combinations of chemotherapy, radiation therapy, or target therapy may be recommended, and many cases can be cure [2]. Unfortunately, the disease is usually diagnosed

\footnotetext{
* Correspondence: li_peng@gibh.ac.cn; yao_yao@gibh.ac.cn

${ }^{2}$ Key Laboratory of Regenerative Biology, South China Institute for Stem Cell Biology and Regenerative Medicine, Guangzhou Institutes of Biomedicine and Health, Chinese Academy of Sciences, Guangzhou 510530, China
} Full list of author information is available at the end of the article in an advanced stage, with a reduced response rate to preceding therapies, which underscores the urgency of discovering new treatment modalities.

Chimeric antigen receptors (CARs) are genetically modified receptors that redirect $\mathrm{T}$ cells to various tumor surface antigens [3]. First generated in the late 1980s and later developed in the early 2010s, a CAR molecule now generally contains an extracellular antigen-binding domain, an intracellular signaling domain, and one or two additional intracellular costimulatory signaling domains $[4,5]$. For clinical use, $T$ cells are harvested from either patients or healthy donors, manipulated to express a specific receptor protein, and then infused into patients after

(c) The Author(s). 2020 Open Access This article is distributed under the terms of the Creative Commons Attribution 4.0 International License (http://creativecommons.org/licenses/by/4.0/), which permits unrestricted use, distribution, and 
expansion. Acting as a living drug, CAR-T cell therapy is rapidly emerging as a promising new treatment for hematological and nonhematological malignancies [6]. In 2017, the FDA approved Novartis's tisagenlecleucel for pediatric B cell precursor acute lymphoblastic leukemia (ALL) and Kite's axicabtagene ciloleucel for adult diffuse large B cell lymphoma [7, 8], thus pushing forward the development of CAR-T cell therapy. Interests have been increased around the possibility that similar success could be achieved in solid tumors. A growing number of preclinical and clinical trials have been conducted ever since [911]. Despite the characterization of EpCAM and claudin as new target antigens in gastric cancer [12, 13], disease heterogeneity remains a substantial obstacle for solid tumor immunotherapy [14], necessitating the identification of alternative antigens.

PSCA, formerly denoted as prostate stem cell antigen, is a glycosylphosphatidylinositol (GPI)-anchored cell surface protein belonging to the Thy- $1 / \mathrm{Ly}-6$ family. This antigen has been recognized as a critical marker in several primary cancers, including bladder, prostate, and pancreatic cancers [15]. Existing studies have thoroughly acknowledged the availability of utilizing anti-PSCA CAR-T cells in the treatment of metastatic prostate cancer and non-small-cell lung cancer [16, 17]. Specifically, a phase I study employing anti-PSCA CAR-T cells for the treatment of patients with metastatic castrationresistant prostate cancer has recently been initiated. Although extensive research applying this target in multiple malignancies has been carried out, no single study has verified the applicability of targeting PSCA in gastric cancer, irrespective of its proven expression on the gastric cancer cell surface [18].

To address the feasibility of using anti-PSCA CAR-T cells to treat GC, we first confirmed PSCA expression in numerous primary GC tissues and multiple cell lines. $\mathrm{T}$ cells encoding a PSCA-specific CAR exhibited upregulated activation markers and secreted abundant cytokines critical for $\mathrm{T}$ cell immunity upon coculture with BGC-823 cells. Anti-PSCA CAR-T cells also exerted increasing cytotoxicity from low to high E:T ratios. In particular, we showed that the peritumoral application of anti-PSCA CAR-T cells in xenograft GC mouse models imposed efficient tumor control. Altogether, we provided proof-of-principle data for the use of anti-PSCA CAR-T cells in the treatment of gastric cancer.

\section{Methods}

\section{Generation of the anti-PSCA lentiviral vector}

The anti-PSCA scFv fragment was derived from the humanized IG8 anti-PSCA antibody [19] and was synthesized by Genscript Co., Ltd. (NanJing, China) after codon optimization. This fragment was then cloned into a previously reported lentiviral vector containing both CD28 and DAP10 intracellular domains.

\section{Lentivirus production}

To produce lentivirus particles, $293 \mathrm{~T}$ cells cultured in DMEM (Gibco, Life Technologies) were co-transfected with the aforementioned anti-PSCA plasmid together with the packaging constructs psPAX2 and pMD2g using polyethyleneimine (Sigma-Aldrich, St Louis, MO, USA). The supernatant was collected $24 \mathrm{~h}, 48 \mathrm{~h}$, and 72 $\mathrm{h}$ post-transfection and filtered through a $0.45 \mu \mathrm{m}$ filter.

\section{Generation and expansion of CAR-T cells}

PBMCs were extracted from whole blood obtained from healthy donors through Ficoll-Hypaque density gradient centrifugation (Fresenius Kabi Norge, AS, Berg i Østfold, Norway), while primary human $\mathrm{T}$ cells were isolated from PBMCs by means of negative selection with the Pan-T Isolation Kit (Miltenyi Biotec, Germany) and activated by microbeads coated with anti-human CD3, antihuman CD2, and anti-human CD28 antibodies (Miltenyi Biotec, Germany) at a 1:1 ratio for $48 \mathrm{~h}$. Afterwards, T cells were transfected with $293 \mathrm{~T}$ cell supernatant containing lentivirus particles for $6-8 \mathrm{~h}$ in the presence of $8 \mu \mathrm{g} / \mathrm{ml}$ polybrene (Sigma). Two rounds of transduction were conducted, after which $\mathrm{T}$ cells were maintained in RPMI-1640 medium supplemented with 10\% heatinactivated fetal bovine serum (FBS), $300 \mathrm{IU} / \mathrm{ml}$ interleukin-2 (IL-2), and 1\% penicillin/streptomycin.

Peripheral blood mononuclear cells (PBMCs) were generated from healthy donors following informed consent and approved by the Research Ethics Board of Guangzhou Institutes of Biomedicine and Health (GIBH).

\section{Cell lines and reagents}

$293 \mathrm{~T}$ cells were used for lentivirus production and were maintained in DMEM (Gibco, Life Technologies) supplemented with $10 \%$ fetal bovine serum (FBS), $2 \mathrm{mM}$ l-glutamine, $50 \mu \mathrm{M} \beta$-mercaptoethanol, $100 \mathrm{IU} / \mathrm{ml}$ penicillin, and $100 \mathrm{IU} / \mathrm{ml}$ streptomycin. BGC-823 (human gastric adenocarcinoma), KATO III (human gastric carcinoma), and MKN-28 (human gastric carcinoma) cell lines were obtained from IBCB (Shanghai, China) and were cultured in RPMI-1640 complete medium. These cell lines were then transduced with a lentivirus vector coexpressing green fluorescent protein (GFP) and luciferase to generate GLlabeled cells.

\section{Flow cytometry}

Cells were harvested and suspended in $50 \mu \mathrm{l} 1 \mathrm{x}$ PBS and primary labeled antibodies were added subsequently. The antibodies used included anti-human PSCA-APC antibody (clone 75) from Santa Cruz Biotechnology 
(Dallas, TX, USA), anti-human CCR7-APC (clone 3D12), anti-human CD62L-PE (clone DREG-56), antihuman CD45RA-APC (clone HI100), anti-human CD45RO-PE (clone UCHL1), anti-human CD27-PE (clone M-T271), anti-human CD25-PE (clone BC96), anti-human CD69-APC (clone FN50), anti-human CD107a-APC (clone H4A3), anti-human CD3-APC (clone UCHT1), anti-human CD4-PerCP/Cy5.5 (clone OKT4), anti-human CD8-PE (clone OKT8), mouse IgG2a isotype control-APC (clone RMG2a-62), and mouse IgG1 kappa isotype control-PE (Biolegend, San Diego, CA, USA). The samples were incubated at $4{ }^{\circ} \mathrm{C}$ for $30 \mathrm{~min}$ before analyzed by NovoCyteTM (ACEA Biosciences), and data were analyzed using FlowJo software (FlowJo, LLC, Ashland, OR, USA).

\section{Cytotoxicity assays}

The cytolytic activity of anti-PSCA CAR-T cells against BGC-823-GL, KATO III-GL, and MKN-28-GL cells was evaluated by incubating the target cells with anti-PSCAexpressing $\mathrm{T}$ cells at the indicated ratio in triplicate wells of white 96-well plates. Target cell viability was monitored $24 \mathrm{~h}$ later by adding $100 \mu \mathrm{l} /$ well D-luciferin (potassium salt) (Yeasen, China) at $100 \mu \mathrm{g} / \mathrm{ml}$. The background luminescence was negligible $(<1 \%$ of the signal from wells containing only target cells). The percentage viability was calculated with the following formula: experimental signal/maximal signal $\times 100$, and the percentage of lysis were normalized to $100 \%$ viability.

\section{Cytokine release assays}

Enzyme-linked immunosorbent assay (ELISA) kits for IL-2, interferon- $\gamma$ (IFN- $\gamma$ ), TNF $\alpha$, and granulocytemacrophage colony-stimulating factor (GM-CSF) were obtained from eBioscience (San Diego, CA, USA), and all ELISAs were performed following the operation manual. T cells were co-cultured with target cells at an E:T ratio of $1: 2$ for $24 \mathrm{~h}$. The culture supernatant was then collected and analyzed for the secretion of IL-2, IFN- $\gamma$, GM-CSF, and TNF $\alpha$ by using ELISA kits.

\section{CDX models for CAR-T cell treatment}

Animal experiments were performed in the Laboratory Animal Center of the Guangzhou Institutes of Biomedicine and Health (GIBH). All procedures were undertaken under the approval of the Institutional Animal Care and Use Committee of GIBH. NOD-SCID-IL2Rg $-/-$ mice were generated as previously described [20]. To develop the cancer xenograft models, NSI mice aged from 6 to 10 weeks were used. All mice were maintained in specific pathogen-free (SPF)-grade cages and provided with autoclaved food and water.

For the BGC-823 cell line-based GC subcutaneous (s.c.) xenograft models, $5 * 10^{5}$ tumor cells suspended in
$150 \mu \mathrm{l}$ PBS were injected subcutaneously into the left flanks of NSI mice. When the tumor nodules were palpable, the mice were treated with $2.5^{*} 10^{6}$ GFP-T cells or anti-PSCA CAR-T cells both by intravenous injection and peritumoral injection. Tumor volume was measured twice a week and was on the track record for over 3 weeks by a vernier caliper. For the MKN-28 subcutaneously transplanted xenograft models, $2.5^{*} 10^{6}$ tumor cells were injected, and $\mathrm{T}$ cells were delivered at a more challenging time point when tumor size went up to around $100 \mathrm{~mm}^{3}$, except which other procedures were followed by the same instructions as described above.

\section{Statistics}

Statistical analysis was performed using GraphPad Prism, Version 7(GraphPad, Inc., San Diego, CA, USA); $p$ values were calculated by unpaired t-test, * indicates $p<0.05$, ** indicates $p<0.01$, and ${ }^{* * * *}$ indicates $p<0.001$.

\section{Results}

PSCA expression in patient tissues and gastric cancer cell lines

To evaluate the potential of the tumor antigen PSCA as an immunotherapeutic target, we immunohistochemically detected its presence and abundance in eight primary gastric cancer samples (Fig. 1a). Most of the analyzed gastric cancer samples expressed PSCA at various frequencies compared to normal tissues. We also performed flow cytometry in several gastric cancer cell lines. The cell types employed in this experiment included BGC-823, MKN-28, and KATO III cells. Uniform expression of PSCA was detected on the surface of these cells (Fig. 1b). Altogether, these data revealed PSCA as a possible novel target for CAR-T cell therapy in GC.

\section{Generation and characterization of anti-PSCA CAR-T cells}

We then constructed a third-generation CAR using a humanized single-chain variable fragment (scFv) derived from a mouse anti-human PSCA antibody and a thirdgeneration lentivirus vector composed of a $\mathrm{CD} 3 \zeta$ intracellular domain and two costimulatory domains, those of CD28 and DAP10, as previously described [20] (Fig. 2a). T cells transfected with only enhanced green fluorescent protein (eGFP) served as the control for unspecific tonic CAR signaling. Primary human $\mathrm{T}$ cells were isolated from peripheral blood mononuclear cells (PBMCs) by magnetic selection and were then activated for $48 \mathrm{~h}$ using CD3/CD28/CD2 beads. CAR expression was detected $48 \mathrm{~h}$ after lentivirus transduction by flow cytometry according to the GFP-positive proportion (Fig. 2b). Transduced $\mathrm{T}$ cells were cultured for 10 days and achieved a final $\mathrm{CD} 45 \mathrm{RO}^{+} \mathrm{CCR} 7^{+} \mathrm{CD} 62 \mathrm{~L}^{\text {high }}$ phenotype (Fig. 2c), implicating their presumed sustainable antitumor potential in vivo. 

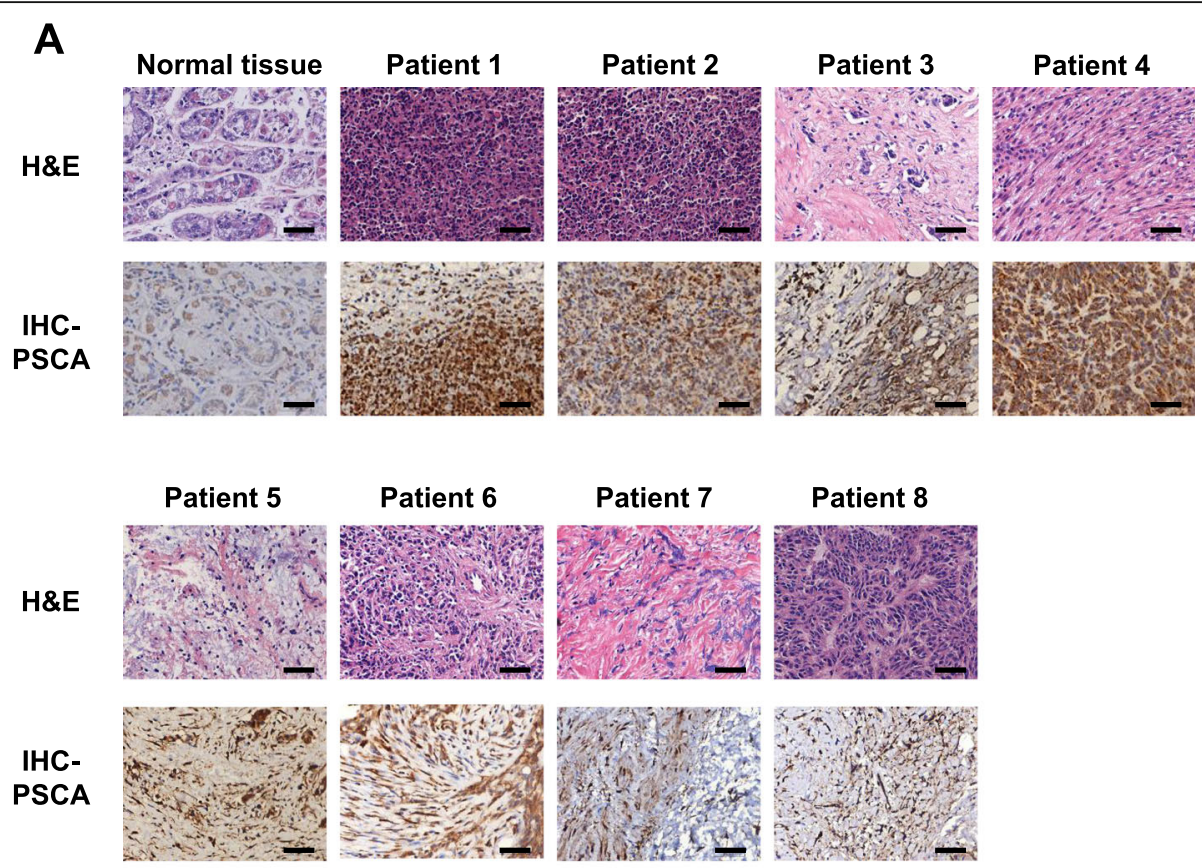

B

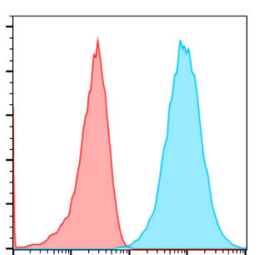

BGC-823

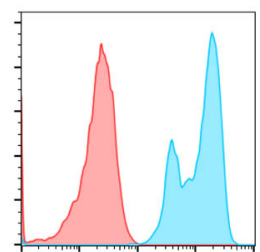

KATO III

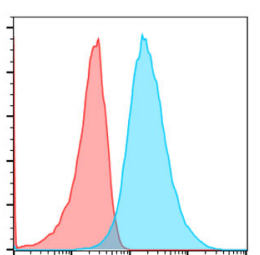

MKN-28

\section{Isotype - PSCA}

Fig. 1 Prostate stem cell antigen (PSCA) expression in primary GC tissues and cell lines. a. Immunohistochemical staining for PSCA in normal gastric tissue and eight primary GC samples; scale bar $=100 \mu \mathrm{m}$. b. Detection of PSCA expression in three human GC cell lines, BGC-823, KATO III, and MKN-28 cells, by flow cytometry

\section{Anti-PSCA CAR-T cells exhibited potent cytotoxicity against $G C$ cell lines}

Next, we sought to evaluate the therapeutic efficacy of anti-PSCA CAR-T cells in vitro. To determine the cytotoxicity of transduced $T$ cells in a more delicate and sensitive way, we genetically modified three target cell lines, BGC-823, MKN-28, and KATO III, to express GFPluciferase. Thus, cell viability could be determined by a luciferase reporter system and an illuminator [21]. We then incubated anti-PSCA CAR-T cells and GFP-T cells with the aforementioned target tumor cell lines at $\mathrm{E}: \mathrm{T}$ ratios of 2:1, 1:1, 1:2, and 1:4. The results showed that anti-PSCA CAR-T cells exhibited more robust cytotoxicity than GFP$\mathrm{T}$ cells after incubation for $24 \mathrm{~h}$ (Fig. 3a). To further investigate the cytokine secretion profile of anti-PSCA CAR-T cells in response to target tumor cells, we collected the culture supernatant from the killing assay described above, and the secreted cytokines were quantified via enzymelinked immunosorbent assay (ELISA). Cytokines, including interferon- $\gamma$ (IFN- $\gamma)$, IL-2, granulocytemacrophage colony-stimulating factor (GM-CSF), and TNF $\alpha$, which are generally secreted by activated $\mathrm{T}$ cells, were examined (Fig. 3b). It was unsurprising to see that anti-PSCA CAR-T cells produced significantly more functional cytokines than control GFP-T cells.

To determine whether anti-PSCA CAR-T cells could be activated by antigen-expressing tumor cells in a target-specific manner, we cultured anti-PSCA CAR-T cells and GFP-T cells with BGC-823 tumor cells at a 1:1 ratio. It has been reported that $\mathrm{T}$ cell activation and differentiation can be monitored based on changes in the expression of a series of surface biomarkers [20]. After coculture with target cells for $24 \mathrm{~h}$, a $\mathrm{T}$ cell surface marker profile was detected by flow cytometry. It was demonstrated that anti-PSCA CAR-T cells were fully activated after coculture with target tumor cells, as indicated by their upregulation of CD25 and CD69 (Fig. 3c, d). Activated anti-PSCA CAR-T cells also downregulated 
A

EGFP

$\begin{array}{llllll}\text { anti-PSCA scFv } & \text { CD28 } & \text { CD3 } & \text { D10 } & \text { 2A } & \text { EGFP }\end{array}$

CD28 Transmembrane Region

B

Non Transduced-T

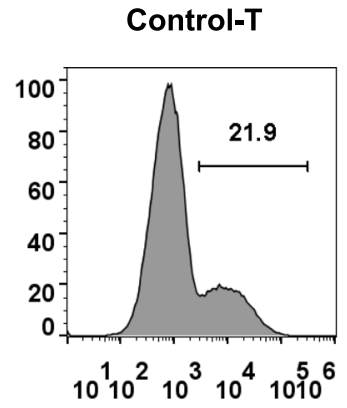

anti-PSCA CAR-T

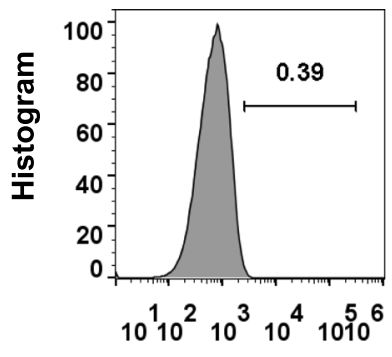

GFP

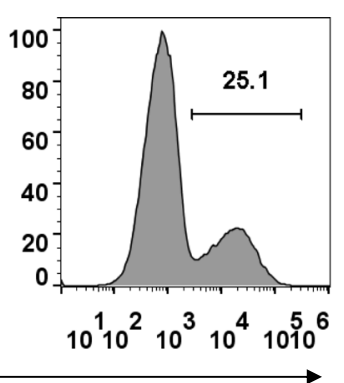

C
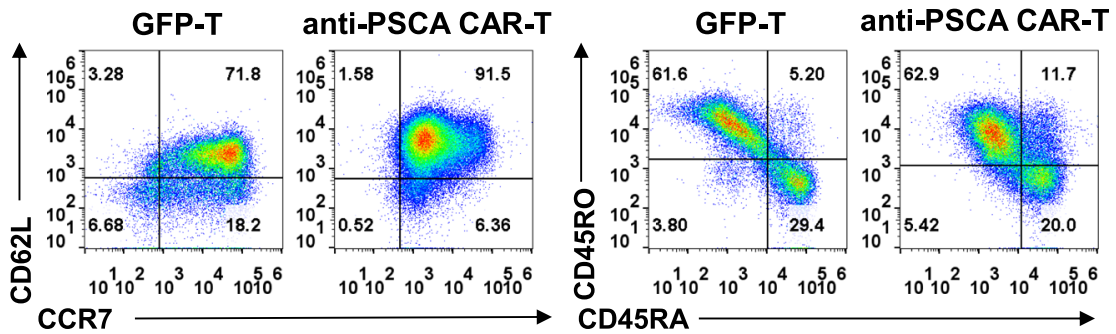

Fig. 2 Generation of anti-prostate stem cell antigen (PSCA) CAR-T cells. a. The discrete CAR units of anti-PSCA CAR-T cells and GFP-T cells. b. Representative flow cytometric analyses of transfected T cells detected by flow cytometry. c. CCR7, CD62L, CD45RA, and CD45RO expression was detected on T cells after their generation

CD62L (Fig. 3c, d), a "homing receptor" that is highly expressed on central memory $\mathrm{T}$ lymphocytes after they encounter antigen but is not found on effector memory T lymphocytes. Moreover, CD27, a costimulatory immune checkpoint molecule, was also downregulated on anti-PSCA CAR-T cells (Fig. 3c, d). Collectively, these experiments demonstrated that CAR-T cells targeting PSCA were fully activated after encountering target tumor cells and exhibited robust cytotoxicity by producing traditional cytokines related to $\mathrm{T}$ cell cytotoxicity.

\section{Anti-PSCA CAR-T cells showed productive antitumor activity in vivo}

Having verified their cytokine release, activation, and cytolytic capacity in vitro, we then sought to evaluate the therapeutic efficacy of anti-PSCA CAR-T cells in vivo by establishing subcutaneous xenograft gastric cancer cell lines in NSI mice. BGC-823 cells were first transplanted, and mice were administered GFP-T cells and anti-PSCA CAR-T cells when Tumor nodules were palpable (Fig. 4a). A previous study validated that peritumoral delivery of CAR-T cells demonstrated improved efficacy compared to intravenous delivery in xenograft models targeting mesothelin [20]. Thus, we used both delivery strategies for each kind of CAR-T cell to further ascertain this phenomenon in our study. Tumor volume was measured twice a week. Compared to the mock group, the GFP-T cell-treated groups, and even the group treated with intravenous anti-PSCA CAR-T cells, the group treated with peritumoral anti-PSCA CAR-T cells showed significant antitumor efficacy (Fig. 4b, c). We also detected the percentage of $\mathrm{T}$ cell infiltration in the tumor, peripheral blood (PB), and spleen (Fig. 4d, e, f). Then, to further validate the cytotoxicity of antiPSCA CAR-T cells in vivo, we established another mouse model by subcutaneously transplanting MKN-28 tumor cells and infused CAR-T cell at a more challenging size of $\sim 100 \mathrm{~mm} 3$, simulating late-stage gastric cancer (Fig. 5a). Anti-CD19 CAR-T cells, universally recognized to eliminate CD19-expressing targets, were used as controls to obviate concerns about the CAR alone. Despite the fact that the heavier tumor burden slightly blunted the CAR-T cell effects, anti-PSCA CAR-T cells still displayed robust antineoplastic capacity when 


\section{A}
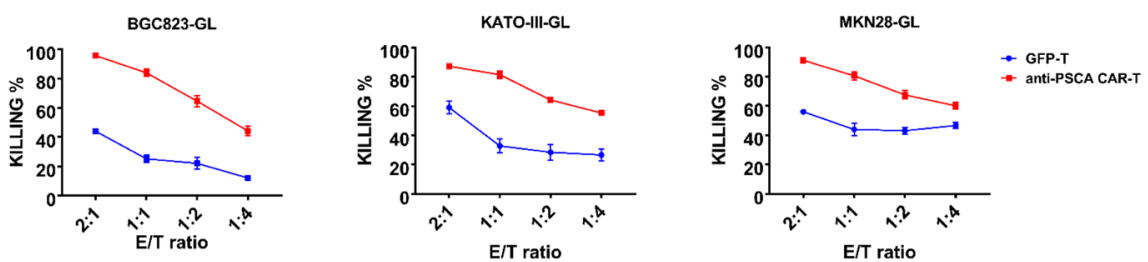

B

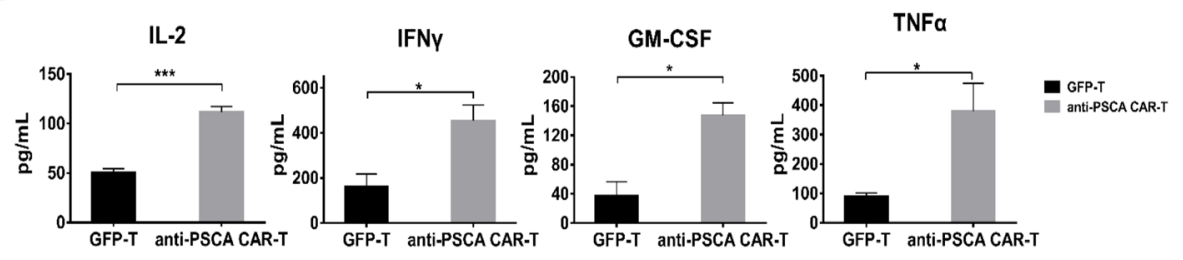

C
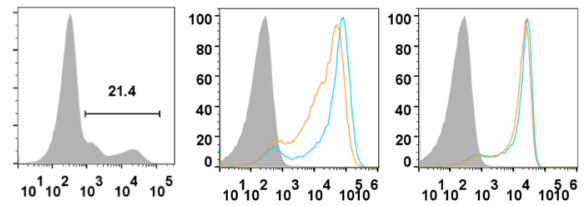

GFP-T
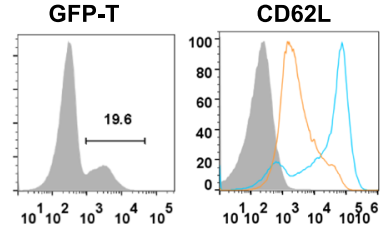

CD27
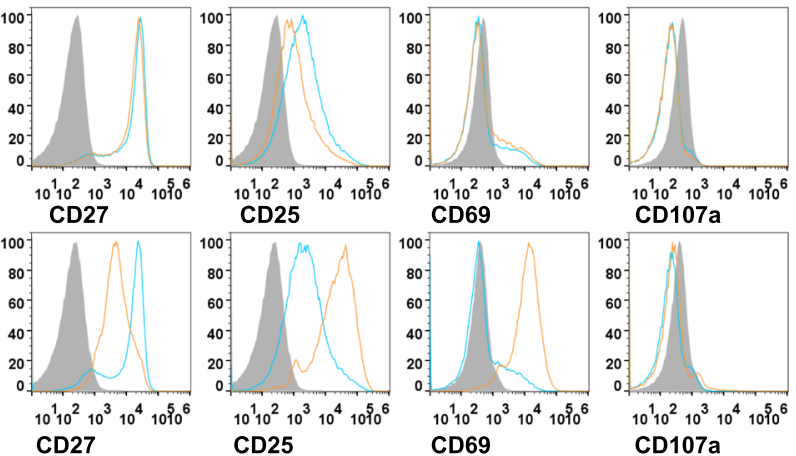
anti-PSCA CAR-T

CD62L

CD27

CD25

CD69

CD107a

Isotype

Before stimulation

After stimulation

\section{D}
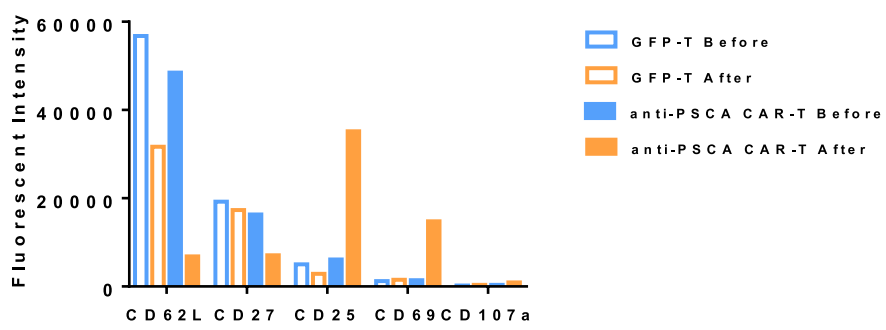

Fig. 3 Anti-PSCA CAR-T cells exhibited dramatic antitumor efficacy ex vivo. a. The lytic capacity towards different target cells, including BGC-823,

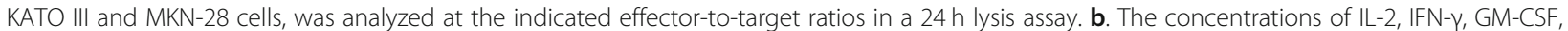
and TNFa released by anti-PSCA CAR-T cells and GFP-T cells after coculture with BGC-823 cells overnight at an E:T ratio of 1:1 are shown. Error bars denote the s.e.m., and the results were calculated by an unpaired t test. ${ }^{*}$ indicates $p<0.05$; ${ }^{*}$ indicates $p<0.01$; and ${ }^{* * *}$ indicates $p<0.001$. c. Canonical T cell markers were detected by flow cytometry at a recommended E:T ratio of 1:1 after coculturing anti-PSCA CAR-T cells and GFPT cells with target cell lines. $\mathbf{d}$. Statistical analysis of three independent FACS results. Error bars denote the s.e.m.

delivered peritumorally (Fig. 5b, c). However, anti-PSCA CAR-T cells transmitted by intravenous injection showed no significant therapeutic ability against PSCAexpressing tumor masses. To address this issue, we examined the $\mathrm{T}$ cell level in different organs after sacrificing the mice. The $\mathrm{T}$ cell infiltration proportion in tumors was not significantly different between the antiPSCA CAR-T cell-treated groups and the control groups (Fig. 5d), regardless of the administration patterns. Only a small proportion of $\mathrm{T}$ cells could be detected in tumor tissues. Further analysis of the peripheral blood (PB) and spleen revealed an increased percentage of $\mathrm{T}$ cells in the 


\section{A}

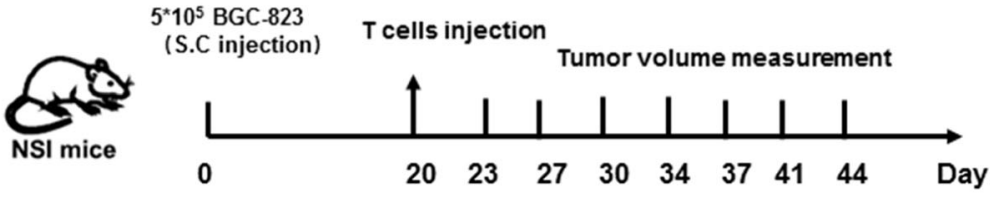

B

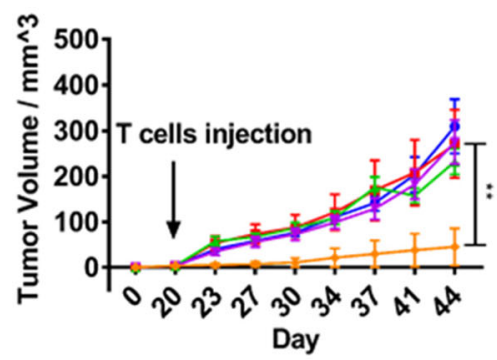

C

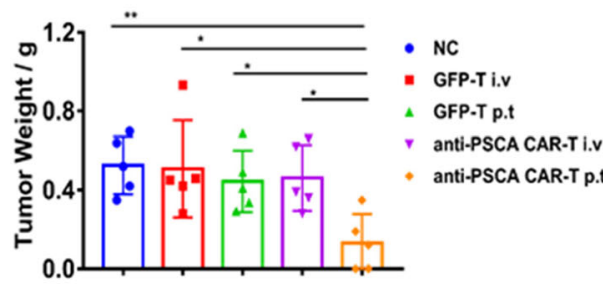

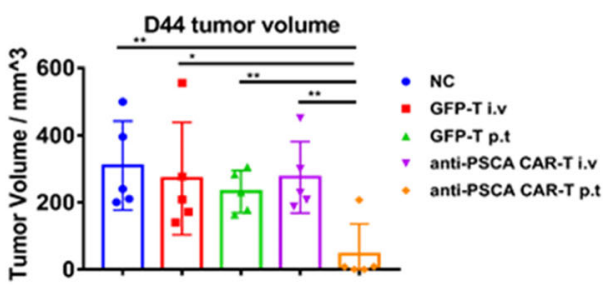

D

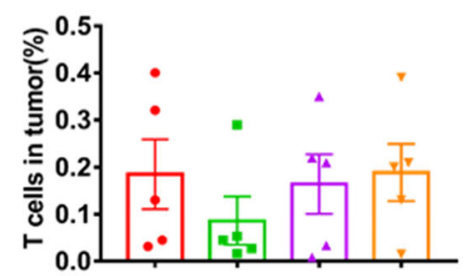

E

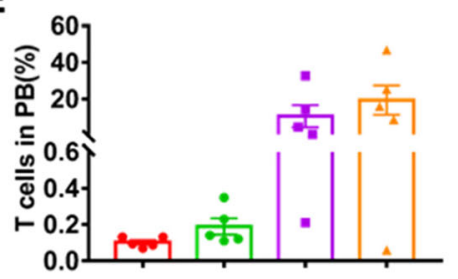

$\mathbf{F}$

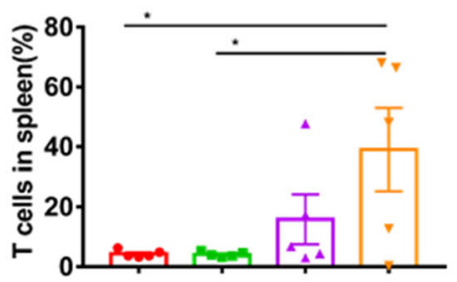

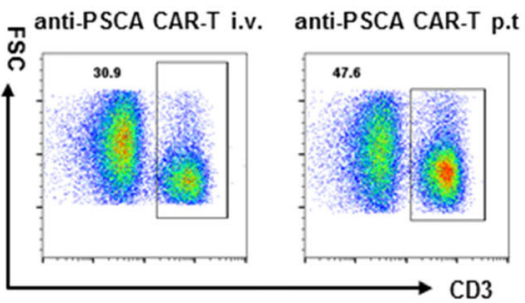

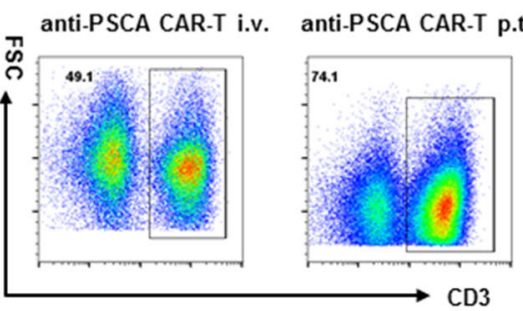

Fig. 4 Anti-PSCA CAR-T cells efficiently reduced tumor progression in BGC-823 models. a. Schematic representation depicting the time course of the experiment. b. Tumor volume was calculated according to the following formula: length $\times$ width $/ 2$. c. Tumor weight of BGC-823 subcutaneously injected mice. Error bars denote the s.e.m., and the results were compared with one-way ANOVA. ${ }^{*} p<0.05 ;{ }^{* *} p<0.01$; ${ }^{* * *} p<$ 0.001. d. Percentage of T cells in the tumor of the BGC-823 models. e. Percentage of T cells in the PB of the BGC-823 models and representative FACS plots of the i.v group and the p.t group, respectively. f. Percentage of T cells in the spleen of the BGC-823 models and representative FACS plots of the i.v group and the p.t group, respectively. Error bars denote the s.e.m., and the results were compared with one-way ANOVA. ${ }^{*} p<0.05$; ${ }^{* *} p<0.01 ;{ }^{* *} p<0.001$

intravenous injection group compared with the peritumorally injected group (Fig. 5e, f), which is slightly different from the performance seen in the BGC-823 model (Fig. 4e, f). In summary, our data demonstrate the remarkable antitumor efficacy of anti-PSCA CAR-T cells in vivo when they are infused peritumorally.

\section{Discussion}

Being the third leading cause of global cancer mortality [22], gastric cancer has gradually imposed a considerable health burden around the world [17, 23]. Traditional treatments such as surgery are only available for early-stage gastric cancer [24], while chemotherapy is usually not recommended 


\section{A}

B
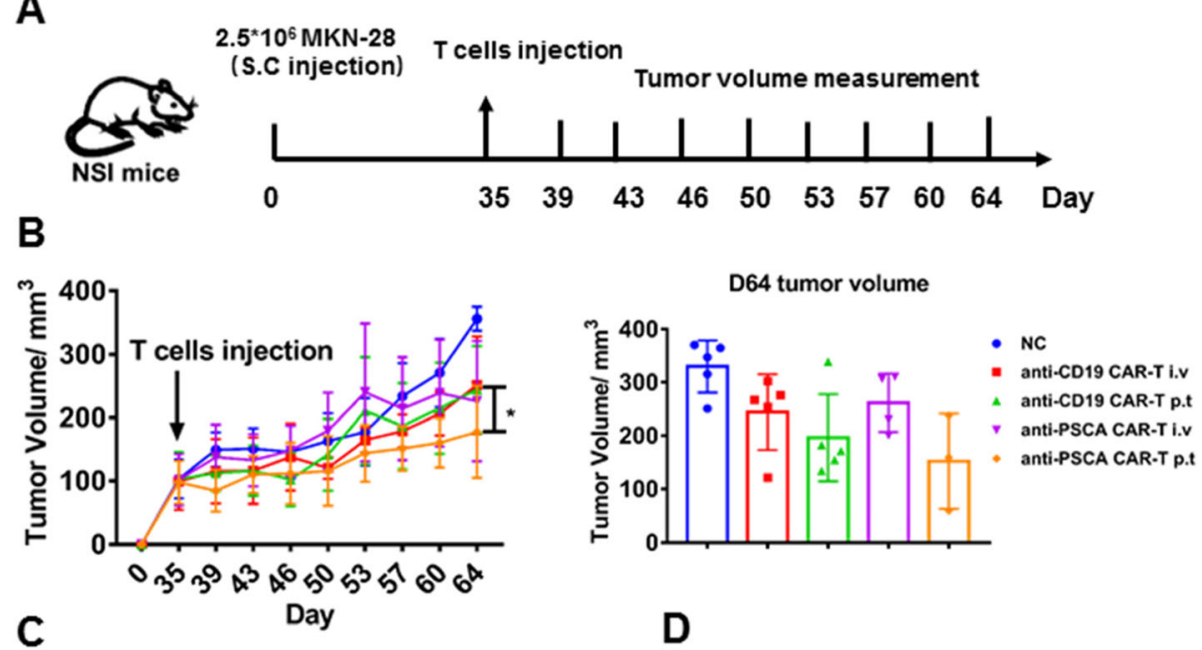

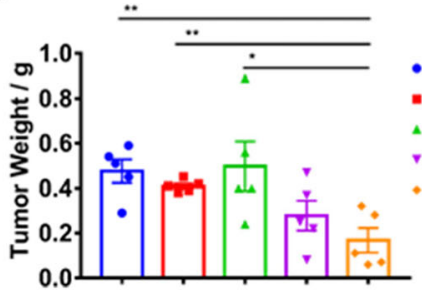

E

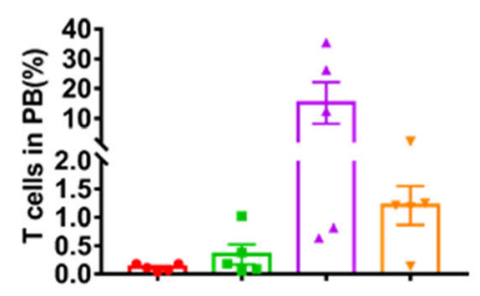

F

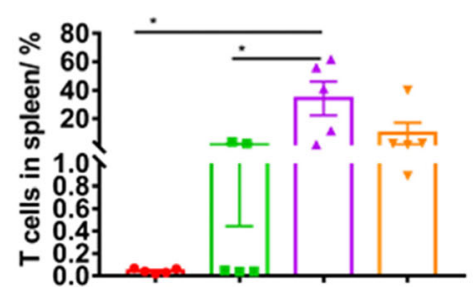

- NC

- anti-CD19 CAR-T i.v

4 anti-CD19 CAR-T p.t

anti-PSCA CAR-T I.V - anti-PSCA CAR-T p.t

D

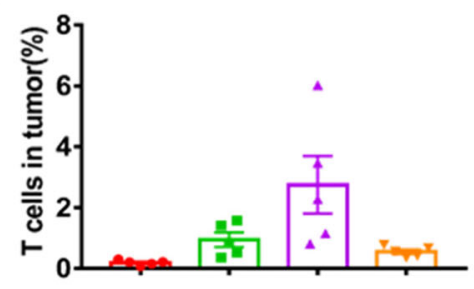

Fig. 5 Anti-PSCA CAR-T cells suppressed tumor progression in MKN-28 models. a. Schematic representation depicting the time course of the experiment. b. Tumor volume was calculated according to the following formula: length $\times$ width ${ }^{2} / 2$. c. Tumor weight of MKN-28 subcutaneously injected mice. Error bars denote the s.e.m., and the results were compared with one-way ANOVA. ${ }^{*} p<0.05 ;{ }^{* *} p<0.01$; ${ }^{* * *} p<0.001$. d. Percentage of T cells in the tumor of the MKN-28 models. e. Percentage of T cells in the PB of the MKN-28 models and representative FACS plots of the i.V group and the p.t group, respectively. f. Percentage of T cells in the spleen of the MKN-28 models and representative FACS plots of the i.v group and the p.t group, respectively. Error bars denote the s.e.m., and the results were compared with one-way ANOVA. ${ }^{*} p<0.05$;

${ }^{* *} p<0.01 ;{ }^{* * *} p<0.001$
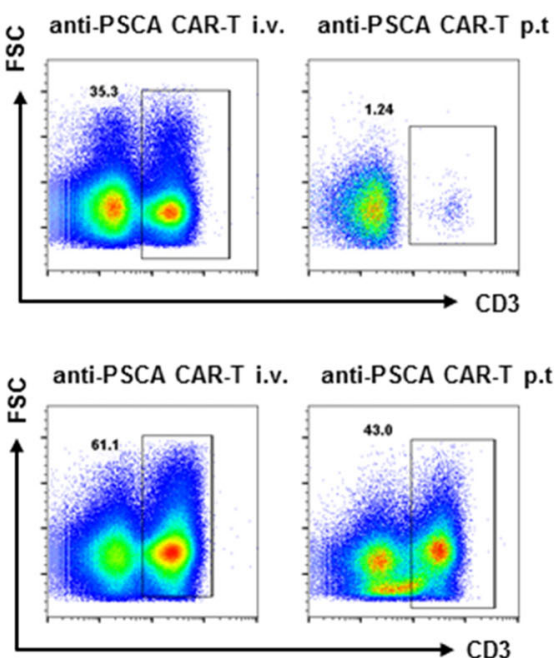

for elderly patients [25], and most patients are not particularly sensitive to these drugs. Targeted therapy has emerged as a promising treatment strategy in recent years, with trastuzumab, targeted at human epidermal growth factor receptor-2 (HER2), and ramucirumab, targeted at vascular 
The advent of CAR-T cell therapy triggered a paradigm shift in cancer immunotherapy [30]. The application of CAR-T cells in hematological malignancies has achieved compelling success [31], sparking considerable interest in in-depth research for this field. Until now, the effects obtained for the treatment of solid tumors have been relatively less encouraging [32]. A potential caveat is posed by the fact that solid tumors are heterogeneous, especially gastric cancer [33]. The lack of truly tumor-specific target antigens is one of the major obstacles. To provide more possibilities for clinical efficacy, we conducted a tentative experiment targeting PSCA in gastric cancer. We constructed a third-generation CAR composed of two costimulatory molecules as previously described [20] and confirmed the potential of PSCA as an ideal antigen for CAR-T cell therapy in gastric cancer. The thirdgeneration CAR we applied herein composed of two costimulatory molecules, CD28 and DAP10. It has been corroborated by our lab that DAP10, the NKG2D associated adaptor protein, is a perfect costimulatory molecule for CAR-T therapy. Incorporation DAP10 in the secondgeneration CAR-T cells could enhance anti-tumor capacity both in vitro and in vivo [34]. We assayed canonical $\mathrm{T}$ cell activation and memory related markers after coculturing anti-PSCA CAR-T cells with target cell lines. AntiPSCA CAR-T cells exhibited upregulated activation markers and cytokine production profiles in an antigendependent manner. Importantly, we demonstrated that anti-PSCA CAR-T cells delivered by peritumoral injection successfully stunted tumor progression in vivo. However, anti-PSCA CAR-T cells given intravenously failed to have any therapeutic effects. Although CAR-T cells given intravenously demonstrated slightly augmented $\mathrm{T}$ cell level in the tumor, peripheral blood, and spleen in $\mathrm{MKN}-28$ cell line based mouse model, there were no statistically significant differences. The absolute number of CAR-T cells remains low, especially within the tumor. Hence, we did not pay much attention to this phenomenon. We then analyzed $\mathrm{T}$ cell population in the tumor, the data showed that CAR positive $\mathrm{T}$ cell percentage moderately increased compared to that of the pre-infused CAR-T cells. It is also possible that there were free PSCA in the blood that masked the CAR-T cells. However, it has been previously reported that CARs can dissociate from dying tumor cells even more rapidly than TCRs [35]. Hence, we did not make it the primary cause of the impaired CAR-T cell cytotoxicity. Much more effort might be taken to investigate whether it is a general phenomenon or a rare case and the mechanism hidden behind. Statistical significance was observed in the tumor weight between the peritumoral injection group and the intravenous injection group in the BGC-823 model, while no differences were seen in the MKN-28 model. Presumably, the augmented peripheral blood and spleen $\mathrm{T}$ cell levels in the peritumoral injection group might have contributed to this deviation. Consistently, these data unveiled another challenge impeding CAR-T cell application in solid tumors, that is, the difficulty of $\mathrm{T}$ cells in migrating to and physically infiltrating into the tumor [36]. As previously reported, much of the current literature on CAR-T cell therapy for solid tumors pays particular attention to T cell trafficking. Kheng Newick (2016) noted that blocking protein kinase A (PKA) localization successfully augmented CAR-T cell trafficking and antitumor efficacy [37]. Along the same lines, Qun Gao (2019) found that DOC induced CD8+ T cell recruitment to the tumor microenvironment by enhancing the secretion of HMGB1 and CXCL11 [38], underlining another view that successful trafficking relies on an appropriate match between the chemokine receptors on T cells and the chemokines secreted by the tumors [39]. The evidence reviewed here seems to suggest a combination strategy, including anti-PSCA CAR-T cells and chemical drugs.

Altogether, our findings corroborated the feasibility and efficacy of anti-PSCA CAR-T cells against gastric cancer, thus providing a new solution for interpatient and intratumor heterogeneity.

\section{Conclusion}

This study identified PSCA as an ideal target for CAR-T cell therapy in GC and achieved impressive efficacy when using third-generation CAR-T cells for GC treatment. The results of this research reveal that anti-PSCA CAR-T cells can specifically eliminate target cells, both ex vivo and in vivo, implicating the potential of antiPSCA CAR-T cells in treating GC patients in the clinic.

\section{Abbreviations \\ ALL: Acute lymphoblastic leukemia; CAR: Chimeric antigen receptor; E:T: Effector to target; ELISA: Enzyme-linked immune absorbance assay; GC: Gastric cancer; GM-CSF: Granulocyte-macrophage colony-stimulating fac- tor; i.p.: Intraperitoneally; i.v.: Intravenous; IFNy: Interferon-y; IL-2: Interleukin-2; p.t.: Peritumoral; p.t.: Peritumoral; PB: Peripheral blood; PBMC: Peripheral blood mononuclear cells mononuclear cells; PBMCs: Peripheral blood; PSCA: Prostate stem cell antigen; s.c.: Subcutaneous; scFv: Single-chain variable fragment}

\section{Acknowledgments}

We would like to thank all the members in the list for experimental materials, technical assistance, helpful discussions, and comments.

\section{Authors' contributions \\ Conception and design: DW, PL, YY. Development of methodology: DW, JL, RZ, ZW, DZ, JS. Acquisition of data (provided animals, acquired and managed patients, provided facilities, etc.): DW, JL, RZ, QW, SL, YL, SW, PL, YY. Analysis and interpretation of data (e.g., statistical analysis, biostatistics, computational analysis): DW, JL, RZ, YL, PL, YY. Writing, review, and/or revision of the manuscript: DW, JL, RZ, PL, YY. Administrative, technical, or material support (i.e., reporting or organizing data, constructing databases): YY, PL. Study supervision: $\mathrm{PL}, \mathrm{YY}$. All authors read and approved the final manuscript.}

\section{Funding}

This study is supported by the National Natural Science Foundation of China (NSFC), No. 81961128003 ; The Strategic Priority Research Program of the Chinese Academy of Sciences, Grant No. XDB19030205; Guangdong 
Provincial Significant New Drugs Development, No. 2019B020202003; The National Major Scientific and Technological Special Project for "Significant New Drugs Development", No. SQ2018ZX090201; Guangdong Provincial Applied Science and Technology Research\& Development Program, No. 2016B020237006; Frontier Research Program of Guangzhou Regenerative Medicine and Health Guangdong Laboratory, No. 2018GZR110105003; Science and Technology Planning Project of Guangdong Province, China (2017B030314056)

\section{Availability of data and materials}

All data generated or analyzed are available from the corresponding author on reasonable request.

\section{Ethics approval and consent to participate}

Healthy PBMCs providers, donors provided gastric cancer tissues and normal tissues, have given consent. All animal experiments were performed at the Laboratory Animal Center of the Guangzhou Institutes of Biomedicine and Health $(\mathrm{GIBH})$. All procedures were approved by the Research Ethics Board of Guangzhou Institutes of Biomedicine and Health (GIBH).

\section{Consent for publication}

Not applicable.

\section{Competing interests}

The authors declare that they have no competing interests.

\section{Author details}

'School of Life Sciences, University of Science and Technology of China, Hefei 230027, China. ${ }^{2}$ Key Laboratory of Regenerative Biology, South China Institute for Stem Cell Biology and Regenerative Medicine, Guangzhou Institutes of Biomedicine and Health, Chinese Academy of Sciences, Guangzhou 510530, China. ${ }^{3}$ Guangdong Provincial Key Laboratory of Stem Cell and Regenerative Medicine, South China Institute for Stem Cell Biology and Regenerative Medicine, Guangzhou Institutes of Biomedicine and Health, Chinese Academy of Sciences, Guangzhou 510530, China. ${ }^{4}$ University of Chinese Academy of Sciences, Shijingshan District, Beijing 100049, China. ${ }^{5}$ Institute of Hematology, Medical College, Jinan University, Guangzhou 510632, China. ${ }^{6}$ Hefei Institute of Stem Cell and Regenerative Medicine, Guangzhou Institutes of Biomedicine and Health, Chinese Academy of Sciences, Guangzhou 510530, China.

\section{Received: 5 November 2019 Accepted: 2 January 2020}

\section{Published online: 28 January 2020}

\section{References}

1. Crew KD, Neugut Al. Epidemiology of gastric cancer. World J Gastroenterol: WJG. 2006;12(3):354.

2. Correa P, Piazuelo MB, Camargo MC. The future of gastric cancer prevention. Gastric Cancer. 2004;7(1):9-16.

3. Zhao J, Lin Q, Song Y, Liu D. Universal CARs, universal T cells, and universal CAR T cells. J Hematol Oncol. 2018;11(1):132.

4. Chang ZL, Chen YY. CARs: synthetic immunoreceptors for cancer therapy and beyond. Trends Mol Med. 2017;23(5):430-50.

5. Zhang C, Liu J, Zhong JF, Zhang X. Engineering car-t cells. Biomark Res. 2017:5(1):22

6. Newick K, Moon E, Albelda SM. Chimeric antigen receptor T-cell therapy for solid tumors. Mol Ther-Oncol. 2016;3:16006.

7. Shum T, Kruse RL, Rooney CM. Strategies for enhancing adoptive T-cell immunotherapy against solid tumors using engineered cytokine signaling and other modalities. Expert Opin Biol Ther. 2018;18(6):653-64.

8. Wang Z, Han W. Biomarkers of cytokine release syndrome and neurotoxicity related to CAR-T cell therapy. Biomark Res. 2018;6:4.

9. Yu S, Li A, Liu Q, Li T, Yuan X, Han X, Wu K. Chimeric antigen receptor T cells: a novel therapy for solid tumors. J Hematol Oncol. 2017;10(1):78.

10. Li J, Li W, Huang K, Zhang Y, Kupfer G, Zhao Q. Chimeric antigen receptor T cell (CAR-T) immunotherapy for solid tumors: lessons learned and strategies for moving forward. J Hematol Oncol. 2018;11(1):22.

11. Li Z, Song W, Rubinstein M, Liu D. Recent updates in cancer immunotherapy: a comprehensive review and perspective of the 2018 China Cancer immunotherapy workshop in Beijing. J Hematol Oncol. 2018; 11(1):142.
12. Wenqi D, Li W, Shanshan C, Bei C, Yafei Z, Feihu B, Jie L, Daiming F. EpCAM is overexpressed in gastric cancer and its downregulation suppresses proliferation of gastric cancer. J Cancer Res Clin Oncol. 2009;135(9):1277-85.

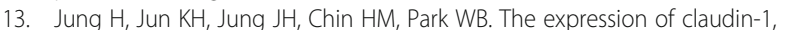
claudin-2, claudin-3, and claudin-4 in gastric cancer tissue. J Surg Res. 2011; 167(2):e185-91.

14. Padmanabhan N, Ushijima T, Tan P. How to stomach an epigenetic insult: the gastric cancer epigenome. Nat Rev Gastroenterol Hepatol. 2017;14(8):467.

15. Saeki N, Gu J, Yoshida T, Wu X. Prostate stem cell antigen: a Jekyll and Hyde molecule? Clin Cancer Res. 2010;16(14):3533-8.

16. Priceman SJ, Gerdts EA, Tilakawardane D, Kennewick KT, Murad JP, Park AK, Jeang B, Yamaguchi Y, Yang X, Urak R. Co-stimulatory signaling determines tumor antigen sensitivity and persistence of CAR T cells targeting PSCA+ metastatic prostate cancer. Oncoimmunology. 2018;7(2):e1380764.

17. Wei X, Lai Y, Li J, Qin L, Xu Y, Zhao R, Li B, Lin S, Wang S, Wu Q. PSCA and MUC1 in non-small-cell lung cancer as targets of chimeric antigen receptor T cells. Oncoimmunology. 2017;6(3):e1284722.

18. Zhao X, Wang F, Hou M. Expression of stem cell markers nanog and PSCA in gastric cancer and its significance. Oncol Lett. 2016;11(1):442-8.

19. Olafsen T, Gu Z, Sherman MA, Leyton JV, Witkosky ME, Shively JE, Raubitschek AA, Morrison SL, Wu AM, Reiter RE. Targeting, imaging, and therapy using a humanized antiprostate stem cell antigen (PSCA) antibody. J Immunother. 2007;30(4):396-405.

20. Lv J, Zhao R, Wu D, Zheng D, Wu Z, Shi J, Wei X, Wu Q, Long Y, Lin S. Mesothelin is a target of chimeric antigen receptor $T$ cells for treating gastric cancer. J Hematol Oncol. 2019;12(1):18.

21. Corso S, Isella C, Bellomo SE, Apicella M, Durando S, Migliore C, Ughetto S, D'Errico L, Menegon S, Moya-Rull D. A comprehensive PDX gastric cancer collection captures cancer cell intrinsic transcriptional MSI traits. Cancer Res. 2019:2019:1166.

22. Wadhwa R, Song S, Lee J-S, Yao Y, Wei Q, Ajani JA. Gastric cancer-molecular and clinical dimensions. Nat Rev Clin Oncol. 2013;10(11):643.

23. Liu D, Mehta D, Kaur S, Kumar A, Parikh K, Chawla L, Patel S, Devi A, Saha A. Decreasing mortality and hospitalizations with rising costs related to gastric cancer in the USA: an epidemiological perspective. J Hematol Oncol. 2018; 11(1):138.

24. Jeong O, Park Y-K. Clinicopathological features and surgical treatment of gastric cancer in South Korea: the results of 2009 nationwide survey on surgically treated gastric cancer patients. J Gastric Cancer. 2011;11(2):69-77.

25. Kim HS, Kim JH, Kim JW, Kim BC. Chemotherapy in elderly patients with gastric cancer. J Cancer. 2016;7(1):88.

26. Roukos DH. Targeting gastric cancer with trastuzumab: new clinical practice and innovative developments to overcome resistance. Ann Surg Oncol. 2010;17(1):14-7.

27. Casak SJ, Fashoyin-Aje I, Lemery SJ, Zhang L, Jin R, Li H, Zhao L, Zhao H, Zhang $H$, Chen $H$. FDA approval summary: ramucirumab for gastric cancer. Clin Cancer Res. 2015;21(15):3372-6.

28. Meza-Junco J, Au H-J, Sawyer MB. Critical appraisal of trastuzumab in treatment of advanced stomach cancer. Cancer Manag Res. 2011;3:57.

29. Fusco N, Rocco EG, Del Conte C, Pellegrini C, Bulfamante G, Di Nuovo F, Romagnoli S, Bosari S. HER2 in gastric cancer: a digital image analysis in pre-neoplastic, primary and metastatic lesions. Mod Pathol. 2013;26(6):816.

30. Liu D, Zhao J, Song Y. Engineering switchable and programmable universal CARs for CAR T therapy. J Hematol Oncol. 2019;12(1):69.

31. Zhao J, Song Y, Liu D. Clinical trials of dual-target CAR T cells, donor-derived CAR T cells, and universal CAR T cells for acute lymphoid leukemia. J Hematol Oncol. 2019;12(1):17.

32. Li D, Li X, Zhou W-L, Huang Y, Liang X, Jiang L, Yang X, Sun J, Li Z, Han WD. Genetically engineered T cells for cancer immunotherapy. Signal Transduction Targeted Ther. 2019;4(1):1-17.

33. Patil DT, Rubin BP. Genetics of gastrointestinal stromal tumors: a heterogeneous family of tumors? Surg Pathol Clin. 2015;8(3):515-24.

34. Zhao R, Cheng L, Jiang Z, Wei X, Li B, Wu Q, Wang S, Lin S, Long Y, Zhang X, Wu Y, Du X, Pei D, Liu P, Li Y, Cui S, Yao Y, Li P. DNAX-activating protein 10 co-stimulation enhances the anti-tumor efficacy of chimeric antigen receptor T cells. Oncoimmunology. 2019;8(1):e1509173.

35. Watanabe K, Kuramitsu S, Posey AD Jr, June $\mathrm{CH}$. Expanding the therapeutic window for CAR T cell therapy in solid tumors: the Knowns and unknowns of CAR T cell biology. Front Immunol. 2018;9:2486.

36. Castellarin M, Watanabe $\mathrm{K}$, June $\mathrm{CH}$, Kloss CC, Posey AD. Driving cars to the clinic for solid tumors. Gene Ther. 2018;25(3):165. 
37. Newick K, O'Brien S, Sun J, Kapoor V, Maceyko S, Lo A, Puré E, Moon E, Albelda SM. Augmentation of CAR T-cell trafficking and antitumor efficacy by blocking protein kinase a localization. Cancer Immunol Res. 2016;4(6):541-51.

38. Gao Q, Wang S, Chen X, Cheng S, Zhang Z, Li F, Huang L, Yang Y, Zhou B, Yue D. Cancer-cell-secreted CXCL11 promoted CD8+ T cells infiltration through docetaxel-induced-release of HMGB1 in NSCLC. J Immunother Cancer. 2019;7(1):42

39. Newick K, O'Brien S, Moon E, Albelda SM. CAR T cell therapy for solid tumors. Annu Rev Med. 2017;68:139-52.

\section{Publisher's Note}

Springer Nature remains neutral with regard to jurisdictional claims in published maps and institutional affiliations.

Ready to submit your research? Choose BMC and benefit from:

- fast, convenient online submission

- thorough peer review by experienced researchers in your field

- rapid publication on acceptance

- support for research data, including large and complex data types

- gold Open Access which fosters wider collaboration and increased citations

- maximum visibility for your research: over $100 \mathrm{M}$ website views per year

At BMC, research is always in progress.

Learn more biomedcentral.com/submissions 